\title{
NONLINEAR SIMULATION OF STRESS-STRAIN CURVE OF INFILL MATERIALS USING PLP FIT MODEL
}

\author{
Er. Prajwal Lal Pradhan, Ph.D. \\ Associate. Prof. Civil Engineering Department, Pulchowk Campus, Institute of Engineering, Tribhuvan University \\ Email: prajwal@engineer.com \\ Er. C.V.R. Murty, Ph.D. \\ Prof. Civil Engineering Department, IIT Kanpur, India
}

Karl Vincent Hoiseth, Ph.D.

Prof. Department of Science and Technology, NTNU Norway

Mohan Prasad Aryal, Ph.D.

Prof. Civil Engineering Department, Pulchowk Campus, Institute of Engineering, Tribhuvan University

\begin{abstract}
This paper puts forward an idealization of stress-strain curve of structural materials like bricks, and mortar. In this model, below yield limit, the pattern of the stress-strain relationship is assumed to be linear i.e. modulus of elasticity remains unchanged, whereas beyond the limit, the relationship is supposed to be curvilinear. A quadratic stress function is assumed to formulate the stress-strain curve passing through the points of yield stress $\sigma_{\mathrm{y}}$ and ultimate stress $\sigma_{\mathrm{u}}$. Experimental investigation on the cube-tests of specimen for brick samples and mortar cubes are also presented for the verification of idealized stress-strain relationships.
\end{abstract}

\section{INTRODUCTION}

With the advancement of computational technology and ever going increasing trend of research activities, the demand for inelastic design is increasing day by day. The term inelastic is associated to material whose stress-strain diagram is nonlinear. But, the usual practices follow only the linear stressstrain relationships. The linear relations are acceptable only for the small deformations. Whereas, in most of the cases when the deformation becomes too large, structural members undergo fail before the strains become finite. Such a situation can be frequently observed in infill frame structures. Infill frames are widely constructed using brick masonry infill walls. Since the brick masonry wall possesses highly heterogeneous, non-linear studies are inevitable. Thus, for the analysis of infill frames, the models which can account for nonlinear behavior due to separation of bricks, cracking, bond slip in mortar joints and dowel actions etc., must be used. For this, non-linear characterization of the materials is quite essential to formulate.

These days, engineering practices demand more and more new additional materials. Whose stress-strain behaviors are still not known or they have to be tested in laboratories. For the very common materials like bricks, concrete and mortars, which have a long history, people still are adopting linear relations for stress-strain curve. In the early days, computational challenges and efforts have made people compelled to accept the state of linear assumption. Now the advancement in computational technology stipulates more précised computational techniques, which can minimize the gap between realistic value and the approximation.

Because the laboratory experiment with a higher precision is not always feasible, a 
simplified mathematical simulation and a theory on inelasticity would greatly simplify the problem of nonlinear studies. Keeping in view of computer application with the use of algorithm for easy generation of stress-strain characteristics of the engineering material, this paper introduces an algorithm for the idealization of a non-linear stress-strain curve for different structural masonry materials.

\section{RESEARCH OBJECTIVES}

The current research is carried out to develop an algorithm which can be useful for assessing the idealization of stress-strain of curves of structural materials used in brick masonry infill frame. As stated above, in general case, stress-strain relationship is treated linearly. But, for the nonlinear studies of structural system this is not adequate. To predict the correct stress-strain diagram is easier said than done. Hence, here is a model presented, which deals with idealization of stress-strain curve from the quadratic family. For this, the relation between stress $(\sigma)$ and modulus of elasticity $(E)$ is assumed to be parabolic. The method presented here is called PLP fit model.

\section{PREVIOUS RESEARCH}

From the pioneering work of Pager (1959), people have felt the necessity of the study on non-linear behavior of engineering materials as the strength of a structure depends on the strength of the materials from which it is made [1]. Actual material strengths cannot be known precisely. Structural strengths furthermore depend on the workmanship, the quality of supervision and inspection. Since non-linear solutions are achieved by considering lot of assumptions, which in turn cause the deviation in the actual behavior of the material, the divergence between the actual and the idealized curve should be minimized as far as possible. In this aspect, various attempts have been made to define empirical equations that would fit experimentally observed stress-strain curves.

It is obvious that nonlinear nature of the stress-strain curve is due to the change in modulus of elasticity at every points of strain in stress function. The physical measure of a material to deform under load is termed as modulus of elasticity. It is the ratio of the stress to the strain of a material or combination of materials. Symbolically it is denoted by a letter $E$. It is made up of multiple parameters including the strength of materials used in whole assembly of structure, basically the unit weight of the structure, volume of each component and the materials etc. Even if light weight units are used versus normal weight units, the modulus will be different. Similarly, varying the type of materials of the components or the varying the sizes can also affect the modulus of elasticity.

Plasticity is defined as a permanent deformation of material by the application of stresses which are greater than those necessary to cause yielding of the material [1]. In general it is cumbersome to make exact mathematical model of the stress-strain curve, but some approximations can be made, from which useful conclusions can be drawn. Masonry materials like bricks and mortar in its reality possess inelastic in nature as they can not bear tension. In order to solve the nonlinear problems, it is necessary to make some assumptions. The first is that the material is isotropic, that is, it has same properties in all directions and it remains isotropic during plastic deformation. Second assumption is that the material obeys Hook's law up to the elastic limit, where there is a sharp yield followed by plastic deformation. It is further assumed that during the plastic deformation the rate of straining has no effect on the material. But the formulations based on these assumptions bear only a superficial resemblance to the 
stress-strain curve. Many past researchers have contributed to find the formulation of idealized curve to minimize the deviation between the actual and the idealized curve. In this concern, Ludwig made various attempts to define empirical equations that would fit experimentally observed stress-strain curves. The following is of the Ludwig's formula.

$\sigma=a+b e^{n}$

Where, $a, b$, and $n$ are constants. If $n=1$ the equation represents a rigid constant strain hardening material of yield stress of $a$. If $n<$ 1 then the constant a still equal to the yield stress, but beyond this, curve posses nonlinear. If the constant $a=0$ and $n<1$ then this represents a material that is inelastic from the beginning of the stress-strain curve and exhibit no definite yield point.

Theory of inelasticity can be simplified if simple continuous function can be derived to approximate the stress-strain curve over both the elastic as well as inelastic range [2]. In the case of most of the metals at room temperature, the stress-strain diagram has a finite length which is linear in elastic range followed by the non-linear curve in the inelastic range. It is almost not possible to find a single equation, which will closely approximate the stress-strain curve over the complete range. In this model, the stress strain diagram is approximated by two straight lines describing slope $E$ and slope $\alpha E$, where $E$ is the modulus of elasticity and $\alpha$ is the strain-hardening factor for the material. The intersection of the two straight lines defines the yield stress $\sigma y$ and the yield strain $\varepsilon y$. The two functions representing the stress-strain diagram are:

$\varepsilon \leq \varepsilon_{\mathrm{y}}$ (elastic stress and strain)

$\sigma_{y}=E \varepsilon_{y}$

$E=\frac{\sigma_{y}}{\varepsilon_{y}}$

$E=\frac{\sigma-\sigma_{y}}{\varepsilon-\varepsilon_{y}}$ $\varepsilon \geq \varepsilon_{\mathrm{y}}$ (inelastic stress and strain)

$\sigma=(1-\alpha) \sigma_{y}+\alpha E \varepsilon$

Based on the assumption of a linear relationship between ultimate longitudinal compressive stress and lateral tensile stress of the brick unit, similar formulations were made [3]. Using force equilibrium, i.e. the total lateral tensile stress of the brick is equal to the total compressive force in mortar, and equality of lateral strains in brick and mortar, the formula to predict brickwork strength is obtained. Biaxial tension-compression strength envelopes of brick units of various strengths were established [4]. Further, behavior of mortar was investigated under tri-axial compression.

A single mathematical model for stress-strain relation as shown in equation 6 was formulated by Popovic [5]. Where, ou is the cylindrical strength, $\varepsilon u$ is the ultimate strain and experimental constant $n$. Poisson's ratio is assumed to be constant, unless the material in one of the principle directions has either cracked or crushed. In either of those cases, it is set to be zero. Ultimate strengths in tension and compression are specified and when either is attained the material is assumed to lose its ability to carry load in that direction.

$$
\sigma=\frac{\varepsilon \sigma_{u}}{\varepsilon_{u}} \frac{n}{n-1+\left(\frac{\varepsilon}{\varepsilon_{u}}\right)^{n}}
$$

Further, Naraine and Sinha (1989) gave an exponential relationship represented by the following equation 7 [6]. They found that, this expression for envelope curve remains same for both the cases of loading i.e. loading perpendicular to the bed joint and loading parallel to bed joint. 
$\sigma=\sigma_{u}\left(\frac{\varepsilon}{\mathcal{E}_{u}}\right) e^{1-\frac{\varepsilon}{\varepsilon_{u}}}$

Here, $\sigma u$ and $\varepsilon u$ are the ultimate values of stress and strains.

Yun Ling (1996) has worked on weightedaverage method for determining uni-axial, true tensile stress vs. strain relation after necking is presented for strip shaped samples [7]. The method demands the identification of a lower and an upper bound for the true stress-strain function after necking and expresses the true stress-strain relation as the weighted average of these two bounds. The weight factor is determined iteratively by a finite element model until best agreement between calculated and experimental load extension curves is achieved. Ling's method use the power law, $\sigma=K \varepsilon^{n}$ where $K$ and $n$ are empirical constants determined from known true stress-strain data before necking. This may be useful for extrapolation of the true stress-strain curve beyond necking. But, he has defined the method of weighted average for predicting true stress-strain functions from engineering stress-strain data. The following expression as shown in equation 8 is suitable for reproducing experimental tensile load-extension curves. However this method is not recommended for prediction of fracture strain.

$$
\begin{aligned}
& \sigma=\sigma_{u}\left[\mathrm{w}\left(1+\varepsilon-\varepsilon_{\mathrm{u}}\right)+(1-w) \frac{\varepsilon^{\varepsilon_{u}}}{\varepsilon_{u}^{\varepsilon_{u}}}\right] \\
& \text { where }, 0 \leq w \leq 1
\end{aligned}
$$

Ashok Saxena (1997) has mentioned that often, it is not possible to precisely define the critical stress at which plastic deformation commences, therefore, the operational beyond the yield strength, the material continues to deform plastically until instability is reached [8]. The stress, at which instability occur $\sigma u$ is the ultimate tensile strength and the corresponding strain $\varepsilon u$ is called the ultimate strain. Beyond the ultimate strain, it is concentrated in the region where the neck develops and eventually fracture occurs.

In the same way, Dinesh Panneerselvam et. Al. (2002) have presented a new nonlinear visco-elastic model for the behavior of concrete, which enables to describe concrete's creep and also creep failure and thus its failure envelope [9]. Yuji Kishino (2002) has demonstrated the incremental nonlinearity observed in numerical tests conducted by a discrete element method proposed [10]. Through a series of true triaxial stress-probe tests, he found that the direction of plastic strain increment changes with the component of stress increment that is orthogonal to the current stress and the yield surface normal. The result suggests that the plastic deformation is accompanied by multiple shear mechanisms. In the same way, Wang Tiecheng, Lu Mingi et. Al. (2003) have used tri-axial loading for finding stressstrain relation of concrete material [11]. The method was based on an orthotropic model.

Hualiang Zhong (2006) used a least-squares method to calibrate an exponential model of pig liver based on the assumption of incompressible material under a uni-axial testing mode [12]. With the obtained parameters, the stress-strain curves generated are compared to those from the corresponding model built in ABAQUS and to experimental data, resulting in mean deviations of $1.9 \%$ and $4.8 \%$, respectively.

\section{IDEALIZATION FOR PLP FIT MODEL OF STRESS-STRAIN CURVE}

In the model presented here, it is assumed that modulus of elasticity $E O$ remains constant up to yield limit (Figure 1). As it crosses the yield limit, stress-strain behaves 
nonlinear. The curvilinear path of the curve is idealized in the form of second order polynomial as shown in the equation 9 shown below.

$$
\sigma=a \varepsilon^{2}+b \varepsilon
$$

Where $a, b$ are the constants, which can be determined by the relations shown in equations 10,11 respectively.

$$
\begin{aligned}
& a=\frac{\sigma_{u} \varepsilon_{y}-\sigma_{y} \varepsilon_{u}}{\varepsilon_{u} \varepsilon_{y}\left(\varepsilon_{u}-\varepsilon_{y}\right)} \\
& b=\frac{\sigma_{y}}{\varepsilon_{y}}-\frac{\sigma_{u} \varepsilon_{y}-\sigma_{y} \varepsilon_{u}}{\varepsilon_{u}\left(\varepsilon_{u}-\varepsilon_{y}\right)}
\end{aligned}
$$

Unlike the model of Popovic, Sinha, and Ling $[5,6,8]$ this model does not require any kind of experimental constants. Also, this model does not behave exponential nature of the curve. The simplicity behind this model is that above yield limit, it is assumed that the curve possesses perfectly second order polynomial. And, below the yield point, material behaves perfectly linear up to elastic limit. As the masonry materials like bricks and mortar fails in tension and shear very quickly even before the strain becomes finite, to identify the nonlinear range above yield is too difficult. In this regard, the quadratic model presented (equation 12) in this paper is assumed to be sufficient enough for materials used in masonry structures. In this model, yield stress $\left(\sigma_{y}\right)$, yield strain $\left(\varepsilon_{y}\right)$, ultimate stress $\left(\sigma_{u}\right)$, and ultimate strain $\left(\varepsilon_{u}\right)$, are considered to be known for concerned materials.

$$
\sigma=\sigma_{u}-\left(\sigma_{u}-\sigma_{y}\right)\left(\frac{\varepsilon_{u}-\varepsilon}{\varepsilon_{u}-\varepsilon_{y}}\right)^{2}
$$

Since, above the yield point, the stress-strain curve follows single curve, the modulus of elasticity $E$ for the stress-strain curve can be considered as:

$E=\frac{2\left(\sigma_{u}-\sigma_{y}\right)\left(\varepsilon_{u}-\varepsilon\right)}{\left(\varepsilon_{u}-\varepsilon_{y}\right)^{2}}$

The general expression for the modulus of elasticity $E$ shown above in equation 13 shows that, initial tangent modulus of elasticity $E O$ is two times the secant modulus of elasticity at the yield point. Similarly, when the yield stress $\left(\sigma_{y}\right)$, ultimate stress $\left(\sigma_{u}\right)$, initial tangent modulus of elasticity $\left(E_{0}\right)$, and yield strain $\left(\varepsilon_{y}\right)$, are known, ultimate strain $(\varepsilon u)$ can be estimated as (equation 14):

$\varepsilon_{u}=\frac{2\left(\sigma_{u}-\sigma_{y}\right)}{E_{0}}+\varepsilon_{y}$

Supplementary works have been carried out for the idealization of infill material samples for brick, and mortar as shown in Figure 5 and 6 and verified with the experimental data.

\section{EXPERIMENTAL WORKS}

In the current research work, the stress-strain curves obtained from experimental tests are presented of the materials used in brick masonry infill frames. Some samples (minimum of 10) of bricks and mortars are tested for compressive strength test. With the results thus obtained, idealized stress-strain curves (PLP fit model) are further verified. Tests are conducted for different types of brick specimen, which were collected from different manufacturers and categorized as machine made brick, Harisiddhi bricks, two locally available bricks Local-1 and Local-2; and two traditional old bricks Old- 1 and Old- 
2. The size of the locally available bricks, Harisiddhi and machine made bricks were in an average of $230 \mathrm{~mm}$ length, $110 \mathrm{~mm}$ width and $55 \mathrm{~mm}$ thickness. Whereas the size of traditional old bricks were $210 \mathrm{~mm}$ length, $105 \mathrm{~mm}$ width and $55 \mathrm{~mm}$ thickness. Traditional old bricks were cut into cube of $50 \times 50 \times 50 \mathrm{~mm}^{3}$, such that size factor could be considered in the evaluation of stress-strain results. Old traditional bricks were collected from the old houses which were constructed since at least 2 decades. Ten samples from each category of bricks are tested experimentally for axial compression.

During the testing, in most of the samples, the initial portion of the load-deflection curve shows nonlinearity, which is attributed to slackness in the test frame. Hence, loaddeflection curve is modified updated by shifting origin of the load-deflection curve as shown in Figure. 2. Based on this load deflection data, stress and strain values have been evaluated and fitted into second order polynomial curve to obtain fitted stress-strain curve. On this curve, the value of initial modulus of elasticity $E_{0}$, ultimate strength $f_{u}$ and the ratio $\frac{E_{0}}{f_{u}}$ are computed. Further, average values are calculated for the initial modulus of elasticity $\left(E_{0}\right)_{\text {average }}$, ultimate strength $\left(f_{u}\right)_{\text {average }}$ and the ratio $\left(\frac{E_{0}}{f_{u}}\right)_{\text {average }}$ for each material. Another equivalent average value of initial modulus of elasticity $E_{0}$ is computed by,

$$
\left(E_{0}\right)_{\text {eq.average }}=\left(\frac{E_{0}}{f_{u}}\right)_{\text {average }} .\left(f_{u}\right)_{\text {average }} .
$$

This average $E_{0}$ values is further considered as an initial tangent modulus of elasticity of the concerned material (Table 1). Similar evaluations are adopted for other mortar specimens too. Mortar cubes are tested with the cube size of $50 \times 50 \times 50 \mathrm{~mm} 3$ with the water cement ratio of 0.7 and the mix proportions of 1:3, 1:4 and 1:6 ratios.

\section{COMPRESSIVE STRENGTH TEST}

For the compressive strength test of brick units, tests are performed conforming to the requirements of IS 3495 (Part I) - 1976. The specimens are immersed in water for 24 hours, and then removed from water and air dried. The frogs are filled and flushed with the face of the brick with 1:1 cement and sand mortar. This sample is cured for four days. Then the specimen is placed in a compression-testing machine with flat faces horizontal and the mortar filled face upwards. The load is applied at the rate of 14 $\mathrm{MPa} / \mathrm{min}$ until the brick specimen fails. To obtain the stress-strain curve, average modulus of elasticity, and ultimate strengths, intermediate values of load and deflection are recorded and above mentioned procedures are followed. The fitted stress-strain diagram for different brick samples are shown in Figure 3.

In the case of mortar, ten different samples of mortar in each mortar mixes of 1:3, 1:4 and 1:6 conforming to the requirements of IS: 2250-1981 are tested. Since the bond between mortar and masonry units is largely influenced by initial rate of water absorption of masonry units, 0.7 water cement ratio was adopted in the present study. To prepare the mortar specimens, the cement and sand was mixed in the required proportion properly, then water was added and the mixture was mixed thoroughly until it is of uniform color. The mortar was then placed in the prepared and well-greased mould of the abovementioned size and numbers. The mortar was compacted on the vibrating table. The compacted cube was then kept in room temperature of $27^{\circ} \mathrm{C} \pm 2^{\circ} \mathrm{C}$ for 24 hours. The 
cubes were then removed from the mould and then immersed in clean fresh water for 28 days. After the compressive strength tests, as in the case of bricks, the fitted stress-strain curves of mortar specimens are plotted as shown in Figure 4.

\section{RESULTS}

Experimentally obtained compressive stressstrain diagrams for bricks and mortar are shown in figures 2 and 3. Results show that machine made and Harisiddhi bricks have better performance than the locally available bricks. Scaling down the mortar grade quickly reduces its ultimate compressive strength. Results for average modulus of elasticity, ultimate strength, Poisson's ratio, and yield strengths for both brick and mortar samples are shown in Table 1. Similarly, compressive stress-strain diagrams obtained from both experimental and idealized PLP fit model are plotted in figures 5 and 6 . Looking at the results, it can be observed that the proposed idealized PLP fit model has remarkably high correlation with the curves obtained from experimental investigation. For the verification purpose, stress-strain diagrams are obtained for locally available Local-1 bricks using Popovic model (equation 6), Exponential (equation 7), Bilinear model (equation 2 - 5), PLP Fit model (equation 12); and these models are verified with the experimentally obtained stress-strain diagram (Figure 7). Result showed that Popovic and PLP Fit models have high correlation with experimental results in comparison to Bilinear and Exponential models.

\section{CONCLUSIONS}

It is obvious that the current trend of computational technology demand more reliable data, which further necessitates detailed study on that or the use of reliable simulation tool. In the case of nonlinear studies, either the sufficient experimental data or the efficient idealized models are of very importance. Since the experimental investigations are not cost effective, the models like this might be one of the good alternative tools for generating idealized stress-strain curves of structural materials. In this context, the current model can be a good tool for idealizing stress-strain curves of engineering materials. The current research has simply shown the possibility behind the technique for simulation.

\section{REFERENCES}

[1] Pager, William, An Introduction to Plasticity, 1st Edition, Addison Wesley, Pearson PTR 1959, pp. 148 ISBN 1124128255

[2] James O.Smith, Omar M. Sidebottom, Inelastic Behavior of Load-carrying Members, John Willey \& Sons New York NY USA, ISBN 1124117679, 1965

[3] Francis, A.J., Horman, C.B., and Jerms, L.E., The Effect of Thickness and Other Factor on the Compressive Strength of Brickwork, Proc. @ nd Int. Brick Mas. Conf., Stoke-on-Trent, England, 1970

[4] Khoo, C.L. and Hendry, A.W., Strength Tests on Brick and Mortar under Complex Stresses for the Development of a Failure Criterion for Brickwork in Compression, Bricktish Ceramic Society, Load Bearing Brickwork, No. 21 (1973)

[5] Popovics, S., A Numerical Approach to the Complete Stress-Strain Curve of Concrete, Mag. Concrete Research, Cement \& Concrete Association, V3, 1973, pp. 583-599

[6] Naraine, K. and Sinha, S.N., Behavior of Brick Masonry under Cyclic Compressive Loading, Journal of Structural Engineering, ASCE, 1989

[7] Yun Ling, Uniaxial True Stress-Strain after Necking, AMP Incorporated, AMP Journal of Technology, Vol 5, June 1996 
[8] Ashok Saxena, Non-linear Fracture Mechanics for Engineers, CRC Press, ISBN 0-8493-9496-1, 1997

[9] Dinesh Panneerselvam and Vassilis P. Panoskaltsis, A Nonlinear Viscoelastic Model For Concrete's Creep And Creep Failure, 15th ASCE Engineering Mechanics Conference, Columbia University New York, NY, June 2-5, EM 2002

[10] Yuzi Kishino, The Incremental Nonlinearity Observed in Numerical Tests of Granular Media, 15th ASCE Engineering Mechanics Conference, June 2-5 2002, Columbia University New York, NA
[11] Wang Tiecheng, Lu Mingqi et.al., Stress-strain Relation for Concrete Triaxial Loading, 16th ASCE Engineering Mechanics Conference, June 16-18 2003, University of Washington, Seattle

[12] Hualiang Zhong and Terry M. Peters, Exponential Elastic Model and Its Application in Real-Time Simulation, Imaging Research Laboratories, Robarts Research Institute 100 Perth Drive, London, ON, Canada, N6A 5K8, Medical Imaging 2006: Visualization, Image-Guided Procedures, and Display, edited by Kevin R. Cleary, Robert L. Galloway, Jr., Proc. of SPIE Vol. 6141, 61410Y, 1605-7422/06, 2006 


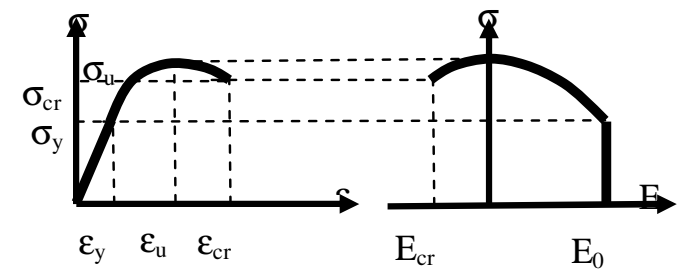

Figure 1: Idealization of stress-strain curve

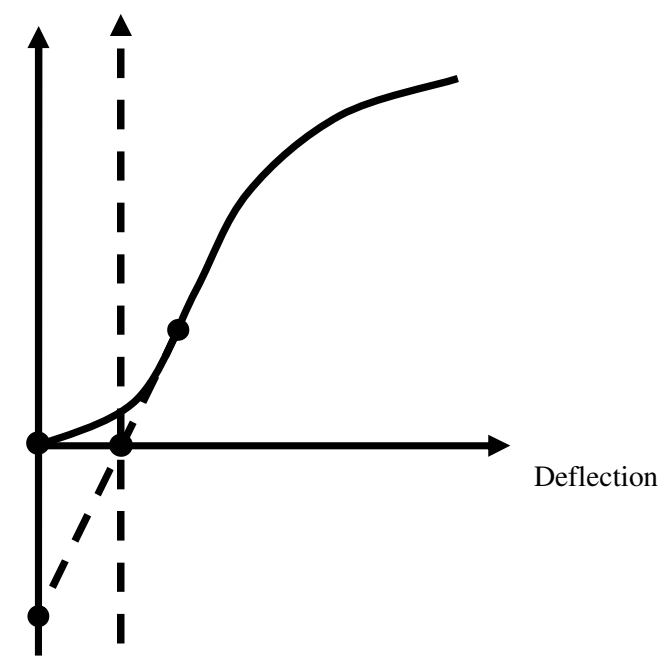

Figure 2: Consideration of strain hardening effect in load deflection curve

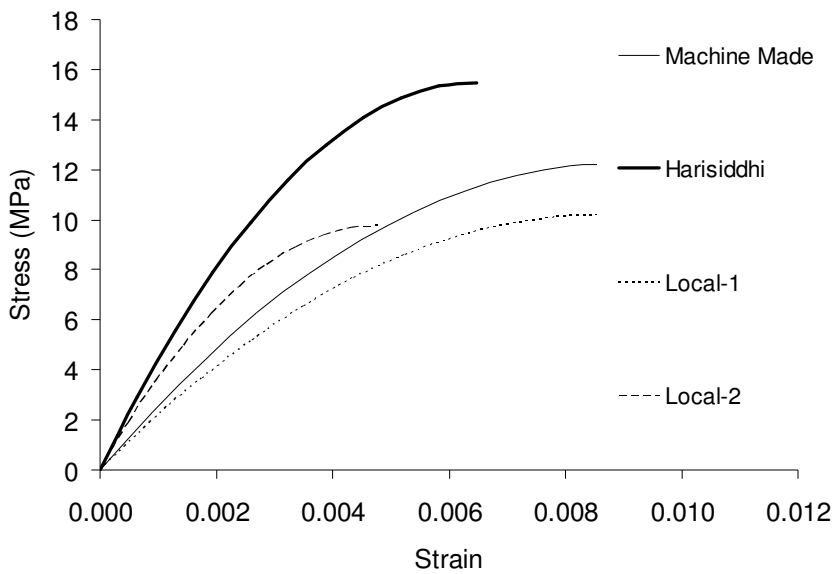

Figure 3: Stress-strain diagram in compression of different types of brick units 


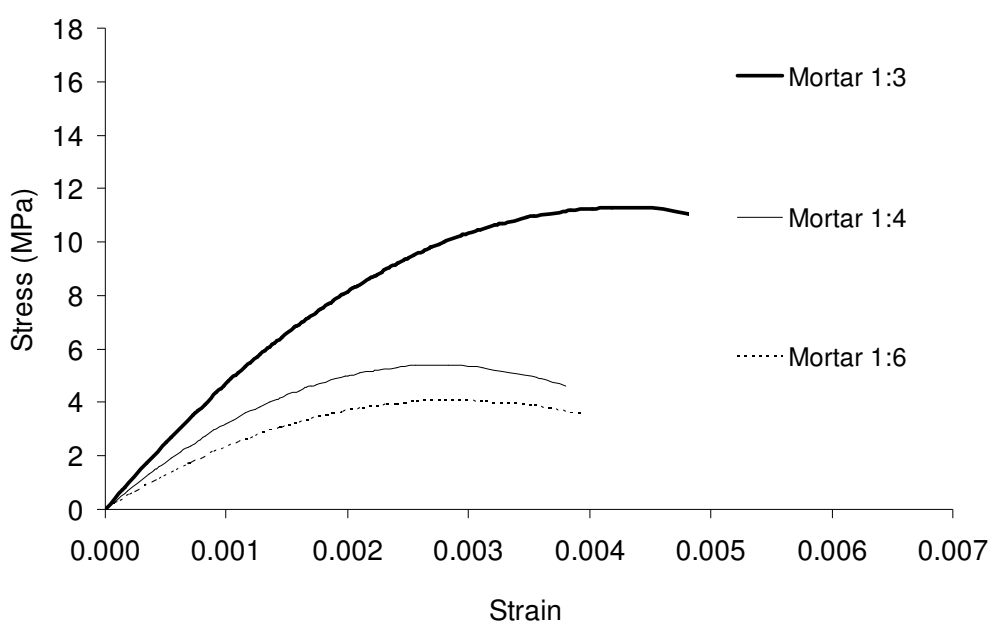

Figure 4: Compressive stress-strain diagram of different mortar mixes
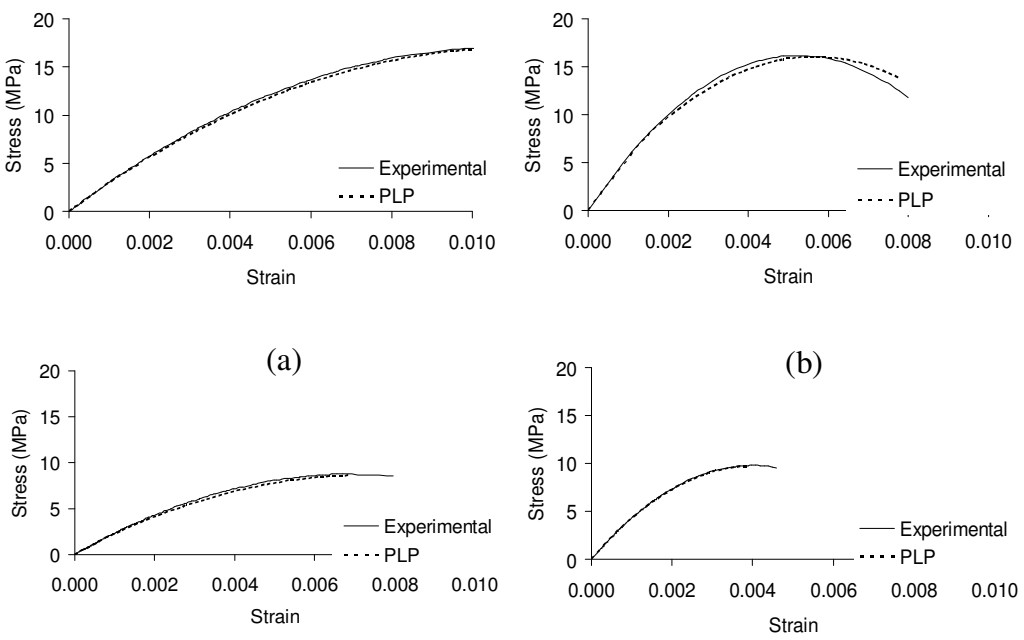

(c)

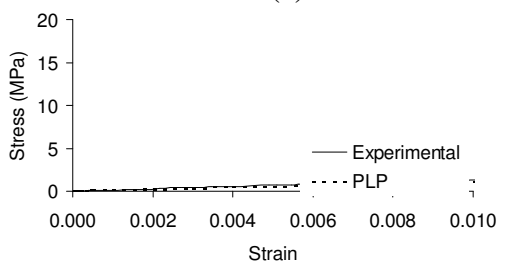

(d)

(e)

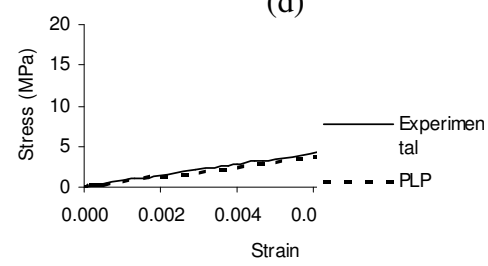

(f)

Figure 5: PLP Fit model of stress-strain curve compared with experimental stress-strain curve for brick samples: (a) Machine made, (b) Harisiddhi, (c) Local -1, (d) Local - 2, (e) Traditional Old - 1, and (f) Traditional Old - 2 


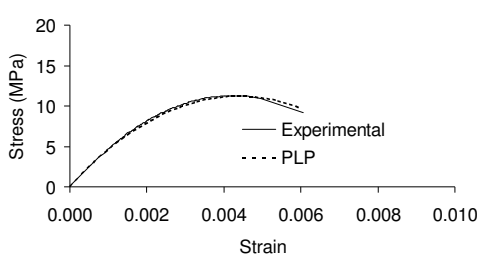

(a)

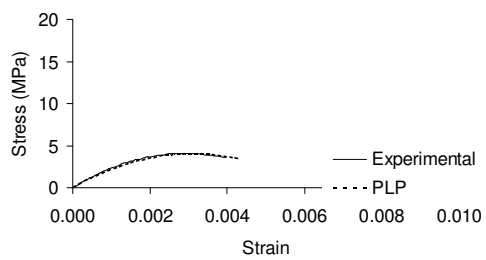

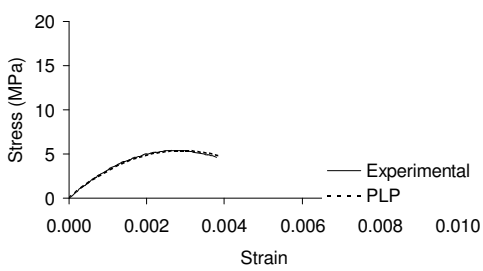

(b)

(c)

Figure 6: PLP Fit model of stress-strain curve compound with experimental stress-strain curve for mortar samples: (a) Mortar 1:3, (b) Mortar 1:4, and (c) Mortar 1:6

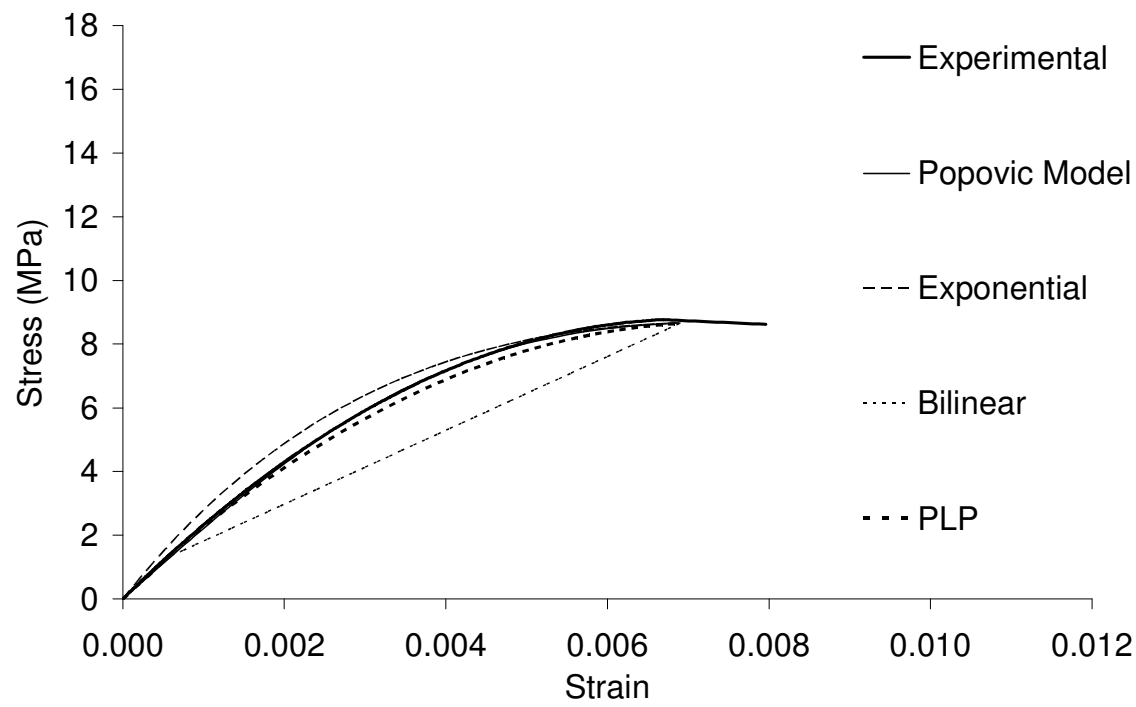

Figure 7: Comparative stress-strain diagram of locally available brick Local-1 
Table 1 Experimental results of specimen test

\begin{tabular}{|l|c|c|c|c|}
\hline \multicolumn{1}{|c|}{$\begin{array}{c}\text { Specimen } \\
\text { type }\end{array}$} & $\begin{array}{c}\text { Modulus } \\
\text { of } \\
\text { Elasticity } \\
\text { (MPa) }\end{array}$ & $\begin{array}{c}\text { Poisson's } \\
\text { Ratio }\end{array}$ & $\begin{array}{c}\text { Yield } \\
\text { Strength } \\
\text { (MPa) }\end{array}$ & $\begin{array}{c}\text { Ultimate } \\
\text { Strength } \\
\text { (MPa) }\end{array}$ \\
\hline Old-1 & 107 & 0.10 & 3.05 & 7.50 \\
\hline Old-2 & 594 & 0.13 & 4.75 & 11.85 \\
\hline Local-1 & 2317 & 0.12 & 1.32 & 8.80 \\
\hline Local-2 & 4576 & 0.14 & 1.25 & 9.11 \\
\hline Machine made & 2938 & 0.11 & 2.55 & 16.97 \\
\hline Harisiddhi & 5365 & 0.09 & 6.44 & 16.01 \\
\hline & & & & 1.7 \\
\hline Mortar 1:3 & 5026 & 0.16 & 0.81 & 5.39 \\
\hline Mortar 1:4 & 3651 & 0.18 & 0.61 & 4.06 \\
\hline Mortar 1:6 & 2616 & 0.14 & & \\
\hline
\end{tabular}

